\title{
MEDIDA DEL GASTO CARDIACO
}

ING. CARLOS ALBERTOS CORTES A. ING. RUBEN DARIO FLOREZ $H$.

Profesores Facultad de Ingeniería de Sistemas Universidad Autónoma de Manizales

\section{INTRODUCCION}

no de los campos más amplios y poco explotados en nuestro medio para realizar investigación aplicada, es el de la Ingeniería Biomédica. En él se pueden enfocar los esfuerzos en la adquisición, tratamiento y análisis de biopotenciales (señales biológicas), al igual que en el diseño, construcción y puesta a punto de equipos y sistemas Software/ Hardware de uso específico en Medicina.

El presente trabajo MEDIDA DEL GASTO CARDiaco es buen ejemplo de ello. Con pocos recursos, y gran dedicación, los autores han pretendido desde el comienzo de su realización presentar un prototipo de bajo costo y versatilidad. respetando los índices de seguridad, robustez y calidad de un sistema tradicional. 
Cuando una persona está en reposo, su corazón bombea entre cuatro (4) y siete (7) litros de sangre cada minuto, sin embargo, durante ejercicio intenso, esa misma persona puede requerir que el bombeo se aumente cuatro (4) y hasta siete veces esa cantidad, con el resultado que el corazón está capacitado para autosintonizarse a tales incrementos extremos en la demanda; esta habilidad intrínseca del corazón de adaptarse a esta dinámica y suplir tal demanda sanguínea es conocida como ley de FRANK - STARLING del corazón (fisiólogos de la segunda mitad del siglo anterior) que enunciada formalmente establece: «Entre mayor sea el llenado del corazón durante la diástole (reposo), mayor será la cantidad de sangre bombeada a la aorta», o sea que el corazón bien puede bombear una pequeña cantidad de sangre o una gran cantidad dependiendo del caudal suministrado por las venas. A esa demanda del flujo sanguíneo por unidad de tiempo se le denomina entonces GASTO CARDIACO.

\section{JUSTIFICACION}

Una de las metas principales en el tratamiento de pacientes con

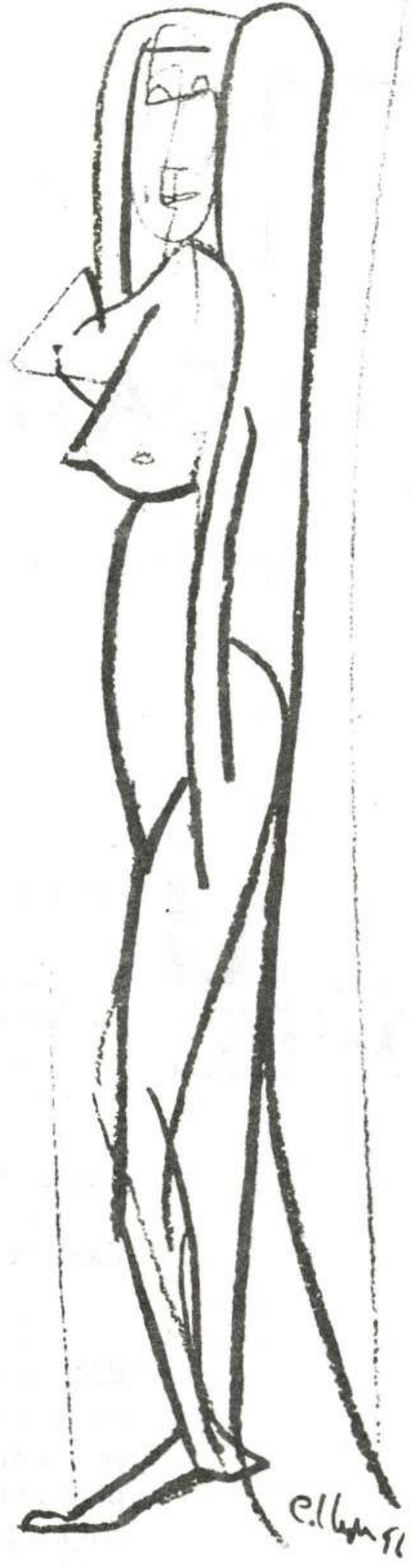


problemas cardiovasculares es satisfacer las necesidades del cuerpo con un mínimo de demandas (esfuerzos) sobre el corazón, resultando entonces de interés medir y analizar diversas variables, una de ellas el GASTO CARDIACO.

\section{OBJETIVOS}

- Determinar el gasto cardíaco en pacientes de la unidad de cuidados intensivos del Hospital de Caldas (UCI).

- Dotar al computador de los elementos y herramientas necesarias (hardware/software) de forma tal que pueda determinar en forma rápida y confiable el Gasto Cardíaco.

- Diseñar, construir y poner en funcionamiento correspondiente sistema de adquisición de datos.

\section{EFECTOS DEL PROYECTO}

\section{Tecnológicos}

Tienen su origen en el marcado incremento tecnológico asociado en su desarrollo en el cual se emplean tecnologías de punta.

\section{Efectos Económicos del Proyecto}

Los costos directos del prototipo 
Medidor del Gasto Cardíaco. son pequeños en comparación con los de un equipo tradicional generalmente importado, además al ser realizado con tecnología propia. su mantenimiento y mejoras constantes quedan al alcance de la mano.

\section{Efectos Sociales del Proyecto}

Al ser un equipo de bajo costo, serían más las clínicas, hospitales y centros de salud que lo tendrian a su disposición, resultando beneficiados más pacientes, aún aquellos de escasos recursos.

\section{ASPECTOS METODOLOGICOS DEL PROYECTO}

\section{Marco Teórico}

En la realización del prototipo fue necesario la aplicación de conceptos de medicina. tecnicas de diseño electrónico, programación. comunicaciones con el computador. traductores.

filtros. acondicionadores de señal e instrumentación electrónica en general.

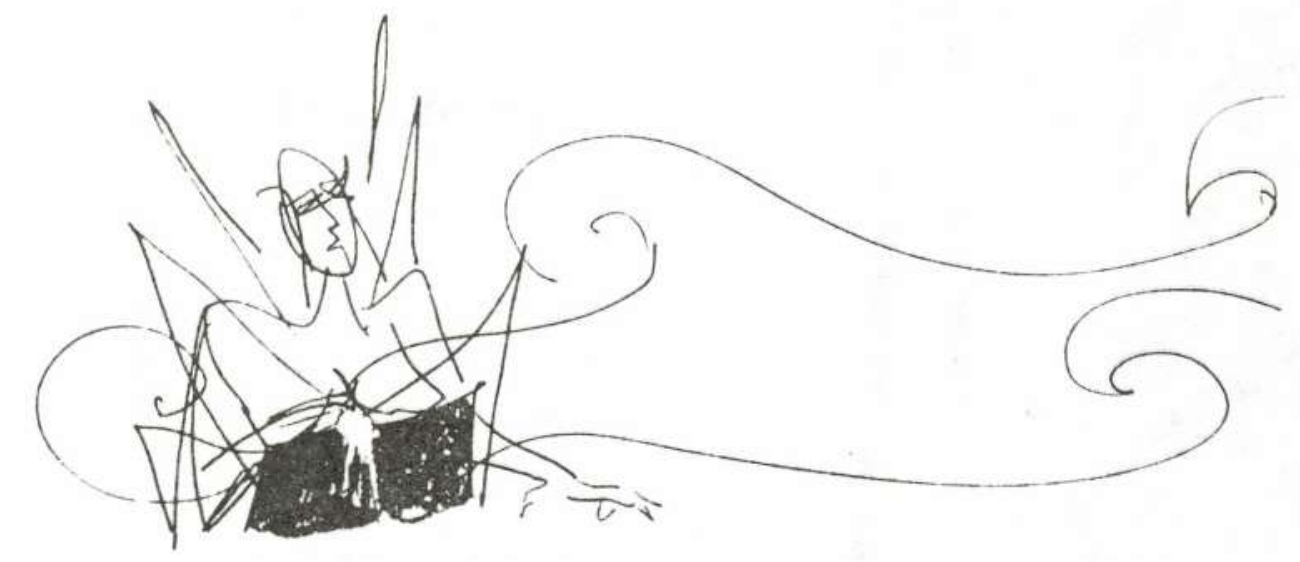



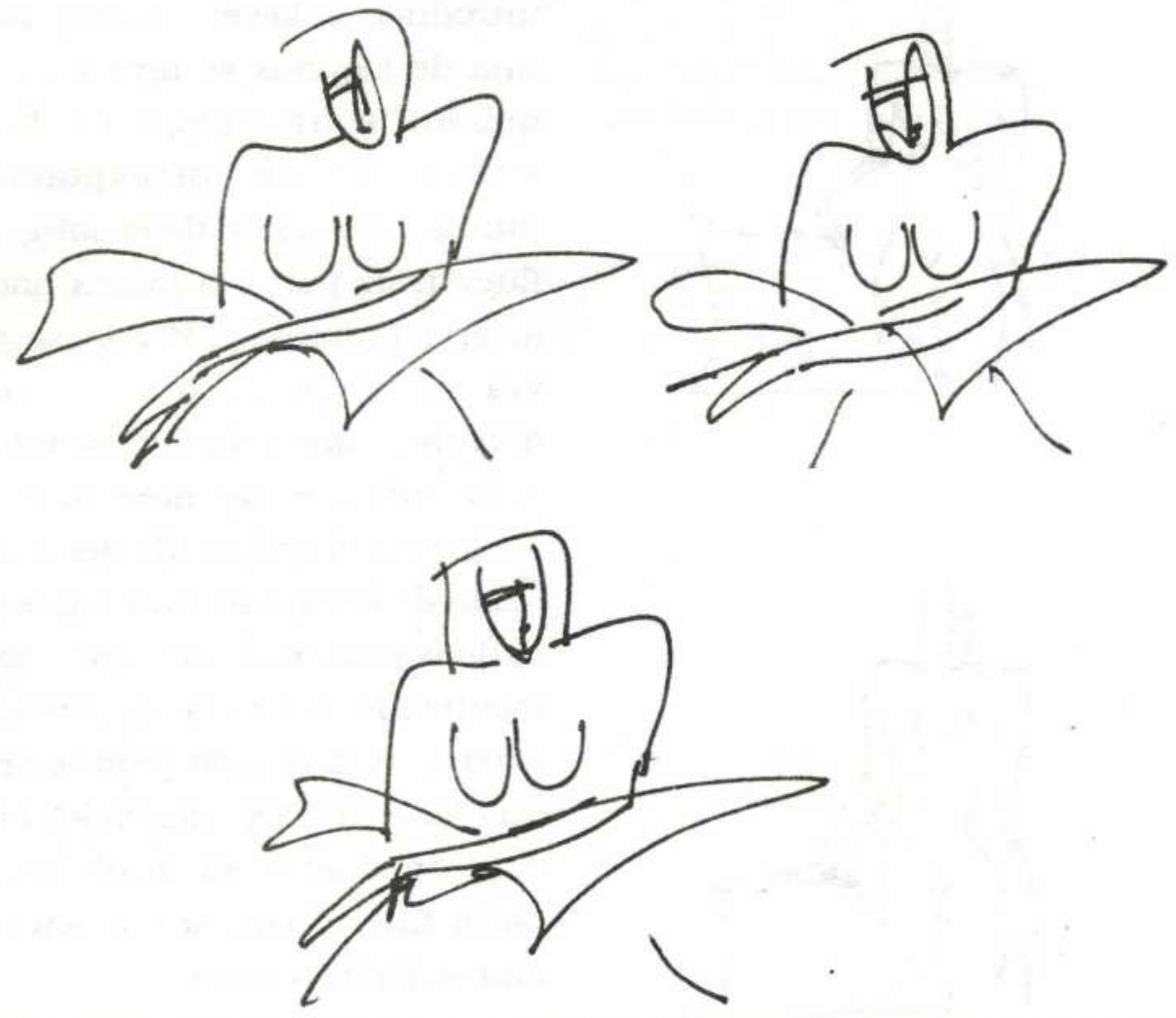

Métodos tradicionales para la medida del Gasto Cardíaco:

Generalmente son técnicas invasivas y las más comunes son:

- Técnica de Fick el indicador es la medida del $\mathrm{O} 2$ con un espirómetro

\section{- Dilución}

- Ultrasonidos

- Termodilución: Es la técnica implementada y se basa en el cuidado y control de la siguiente relación

1.08Ct60V1(Tb-T1)

G. C. $=\frac{\int_{O}^{X} \Delta t b(t) d t}{.08 C t 60 V 1(T b-T 1)}$

G. C.= gasto-cardíaco

$\mathrm{Ct}=$ Calor-solución-inyectada

V1 = Volumen-solución-inyectada

$\mathrm{Tb}=$ Temperatura-inicial-sangre

Tl = Temp. - inicial-solucióninyectada

$1.08=\mathrm{p}$-sangre 

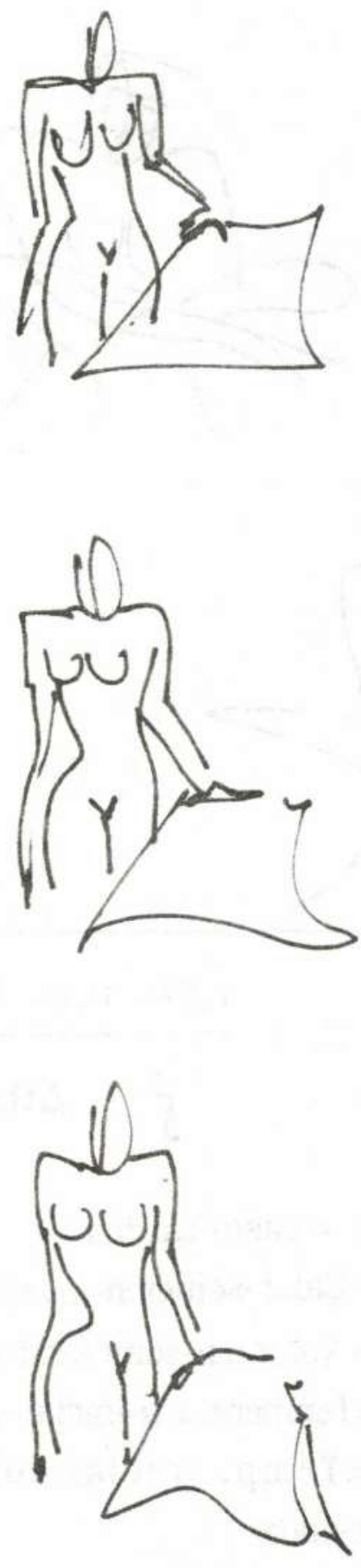

En ésta, un catéter de 4 vías se introduce en la vena branquial. Por una de las vías se inyecta un gas que infla una pequeña bomba situada en su correspondiente punta. La presión de la sangre que fluye transporta la punta hacia la arteria pulmonar. Por la segunda vía se inyecta. a la aurícula derecha, una solución salina fría, solución que se mezcla con la sangre en el ventrículo derecho. La caída de temperatura en la sangre es detectada mediante un termistor localizado cerca a la punta del catéter en la arteria pulmonar, por una tercera vía se tienen los cables del termistor y por la última qque raramente se usa), se pueden tomar muestras de sangre.

El catéter se puede dejar en su lugar sin problemas las 24 horas, tiempo durante el cual se pueden hacer varias mediciones del GASTO CARDIACO, razón por la cual esta forma de medición del G. C. es más ventajosa.

La ecuación simplemente describe el hecho de que dos sustancias a diferentes temperaturas son mezcladas, y que la temperatura resultante es función de la temperatura inicial de las dos sustancias. 
SINTESIS DE LOS RESULTADOS

El prototipo disenado. ha funcionado de acuerdo a lo previsto en su concepción inicial, continúa la etapa de calibración y diseño final.

\section{CAMPO DE APLICACION DE LOS RESULTADOS}

Aunque inicialmente la aplicación está orientada a la medida y cuantificación del gasto cardíaco, la flexibilidad tanto de su hardware como de su software permiten que sea fácilmente aplicable a otros sistemas que requieran captura, digitalización, control y visualización de variables físicas con ayuda del computador.

\section{COMUNICACION DE LOS} RESULTADOS

El prototipo ha sido expuesto ante los médicos de la Unidad de Cuidados Intensivos del Hospital de Caldas de los cuales se recibieron comentarios satisfactorios.

\section{ASPECTOS OPERATIVOS}

\section{Plan de actividades}

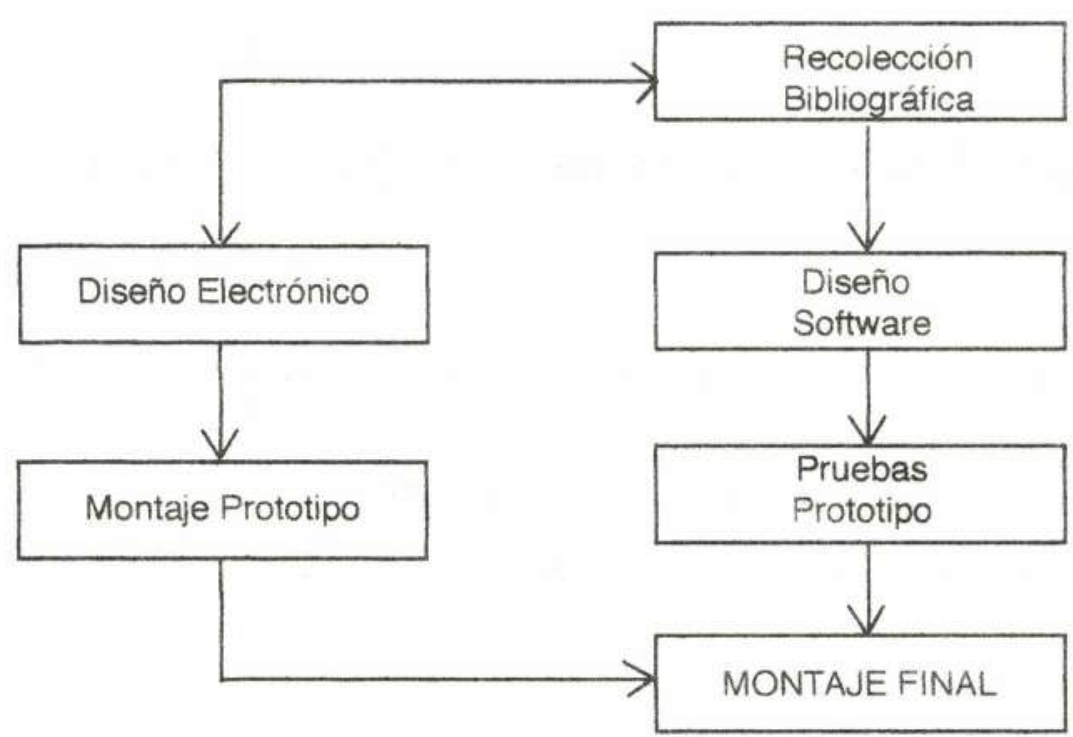


CARACTERISTICAS MEDIDOR

G. C.

El prototipo para la medida del G. C. detecta los cambios de temperatura mediante dos transductores (termistores NTC): tomada la señal. es acondicionadáa. filtrada y manipulada por el computador por medio de un programa que permite su cuantificación y graficación. Las caracteristicas principales son:

\section{DESCRIPCION DEL HARDWARE}

La arquitectura es la correspondiente a la de cualquier sistema de adquisición de datos por computador (ver figura) y consta de las siguientes etapas:
- Transductores: para captar la señal física

- Filtraje y Acondicionador de señal: llevar la senal a niveles manipulables por el computador además de eliminarle impurezas debidas a ruidos e interferencias en general.

- Conversor de Análogo a Digital A/ D: discretizar la señal para llevarla del medio físico al mundo digital que maneja el computador.

\section{DESCRIPCION DEL SOFTWARE}

Se realizó un programa para la captura, cuantificación y visualización de datos en C. (Se anexa ejecutable). $\bigcirc$

\section{DIAGRAMA DE BLOQUES MEDIDOR GASTO CARDIACO}

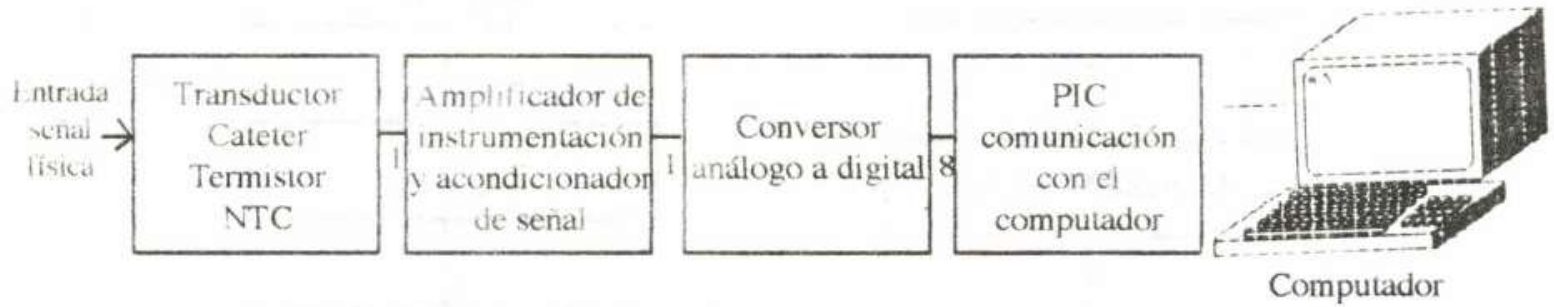

